\title{
Control of Unmanned Helicopter under Disturbance based on Improved Inner-outer Loop Control Strategy
}

\author{
Qin Chuanbo ${ }^{1, a}$, Feng Bao ${ }^{2, b}$ and Fan Hongjun ${ }^{3, c}$ \\ ${ }^{1}$ School of Information Engineering, WuYi University, JiangMen, GuangDong, 529020, China \\ ${ }^{2}$ Department of Electronic Information and Automation, GuiLin University of Aerospace Technology, \\ GuiLin, GuangXi, 541004, China \\ ${ }^{3}$ School of Tourism Management, Hainan College of Economics and Business, HaiKou, HaiNan, \\ 571127, China

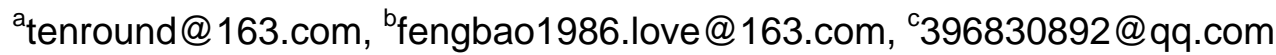

\begin{abstract}
Keywords: Improved PID controller; adaptive back-stepping controller; Attitude control; Unmanned Helicopter
\end{abstract}

Abstract. This paper proposed an inner-outer loop control strategy, which is derived on a simplified non-linear model for unmanned helicopter, to deal with the problem of control stability under disturbances. The outer loop controller, which is an improved PID controller, may adjust PID parameters dynamically to achieve stable control law for position control under disturbance. The inner loop controller is designed for controlling attitude angles under disturbance. By estimating the disturbance beforehand, the inner loop controller may achieve control stability of attitude angles efficiently. Moreover, based on the proposed simplified nonlinear model, design of the Lyapunov function in inner-loop controller can also be simplified, which facilitates engineering application. Numerical experiment results show that the proposed controller can stably control the attitude angle and position of unmanned helicopter under disturbances.

\section{Introduction}

Unmanned helicopter has advantages of more flexible maneuverability (for example: vertically take-off and landing, hovering) so that it is highly favored in the practical work of searching and rescuing, assistant law enforcement, aero-surveying, mapping and so on ${ }^{[1]}$. However, due to the inherent nonlinearities 、 complex dynamic characteristics and instability of the system, design of an efficient and stable controller plays an important role for promoting performance of unmanned helicopter.

Methods of designing unmanned helicopter controller are mainly divided into two categories: linear controller and nonlinear controller. Traditional methods of linear controllers are proportional integral derivative (PID) controller、 linear quadratic regulator (LQR) controller、 linear quadratic gaussian (LQG) controller、 $H-\infty{ }^{[2,3]}$. The main idea of linear controller based methods is to use linear control theory to design the controller based on a linear model, which is designed to approximate the nonlinear process of the unmanned helicopter. Linear controller based methods have been widely used in the area of controlling unmanned helicopter and have achieved great success. However, performance of linear controller based methods may be greatly influenced when the unmanned helicopters worked under unfixed disturbance condition (e.g. wind gust).

Nowadays, nonlinear controller based methods for unmanned helicopters received more and more attention. Traditional nonlinear controller based methods are feedback linearization controller and dynamic inverse controller. However, for the reason of high order and heavy computation burden, traditional nonlinear controller based methods are difficult to be applied in practice. It is still of great value to design a simple and effective nonlinear controlled strategy [4].

To overcome the shortcomings mentioned above, we proposed an improved inner-outer loop control strategy in this paper. The proposed method tracks position and attitude angle under parameter uncertainties and exogenous disturbances situation by combining advantages of improved 
PID controller and robust back-stepping technique. The outer-loop controller is a position controller based on improved PID controller. It takes advantages of tracking position by adjusting control parameters dynamically.

The inner-loop controller is an attitude angle controller based on back-stepping technique [5]. For angle control, stability is an important factor closely related to performance of unmanned helicopter [6]. To achieve anti-disturbance ability, we introduced an attitude angle error term into controlling process to improve performance traditional back-stepping controller. Moreover, by simplifying design of Lyapunov function, the proposed inner-loop controller has flexibility in introducing appropriate dynamics to make the system behave in a more stable way under nonlinear disturbances situation. By combining advantages of both PID and back stepping controllers, the proposed controller not only can work under nonlinear disturbance situation but also can be easily realized in practice.

This paper is organized as follows. In section 2, an overview of the non-linear model of unmanned helicopter is presented. In section 3, the inner-outer loop controller is introduced and designed. In section 4, performance of the controller designed above is evaluated by analyzing the results of simulated experiments. At last, conclusions are given in section 5.

\section{Nonlinear Model of the Unmanned Helicopter}

In general condition, the non-linear system model of unmanned helicopter is a rigid body motion model of six degree of freedom, which can be show as follows:

$$
\begin{array}{ll}
\dot{P} & =V \\
m V & =R_{b g}^{T}(\eta) F^{b} \\
\eta & =H(\eta) \omega^{b} \\
I \dot{\omega}^{b} & =-\omega^{b} \times I \omega^{b}+\tau^{b}
\end{array}
$$

where $F^{b}$ and $\tau^{b}$ are the combined external force and torques respectively, $I$ is the movement inertial matrix, $\mathrm{m}$ is the mass of the UAV. $P=\left[\begin{array}{lll}x & y & z\end{array}\right]^{T}$ and $V=\left[\begin{array}{lll}u & v & w\end{array}\right]^{T}$ are the position and velocity of the unmanned helicopter respectively in the inertial frame. $\omega^{b}=\left[\begin{array}{lll}p & q & r\end{array}\right]^{T}$ represents the helicopter angular rate vector with respect to its body axes. The Euler angle vector $\eta=\left[\begin{array}{llll}\phi & \theta & \psi\end{array}\right]^{T}$ is defined in the roll-pitch-yaw sequence.

Note that when unmanned helicopter performs attitudes of hovering or in a low speed flight, we can ignore the small bonding forces and the resistance of the helicopter body. If we use the symbols $\vec{T}_{M}$ and $\vec{T}_{T}$ to represent the whole thrust from main rotor wing and rear rotor wing respectively, $a$ and b represent the lateral and lengthwise waving angle of the main rotor wing. Denote the thrust vector of the main and tail rotor by $\vec{T}_{M}$ and $\vec{T}_{T}$, we can have the equation as follows:

$$
\vec{T}_{M}=\left[\begin{array}{c}
X_{M} \\
Y_{M} \\
Z_{M}
\end{array}\right]=\left[\begin{array}{c}
-\sin a \cos b \\
\cos a \sin b \\
-\cos a \cos b
\end{array}\right] T_{M} \approx\left[\begin{array}{c}
-a \\
b \\
-1
\end{array}\right] T_{M}
$$

The above equation is simplified based on an assumption that when the helicopter is flying at low speed, the waving angle $a$ and $\mathrm{b}$ are small.

$$
\vec{T}_{T}=\left[\begin{array}{lll}
0 & Y_{T} & 0
\end{array}\right]^{T}=\left[\begin{array}{lll}
0 & -1 & 0
\end{array}\right]^{T} T_{T}
$$

\section{Design of Improved Inner-outer Loop Controller}

The proposed controller is an inner-outer loop control strategy: the outer-loop controller is a position controller based on improved PID controller. The inner loop controller is an attitude angle controller based on robust back-stepping technique. 


\subsection{Outer-loop Controller based on Improved PID}

The traditional PID controller can be described as follows:

$$
u(t)=K_{p}\left[e(t)+\frac{1}{T_{I}} \int e(t) d t+\frac{T_{D} d e(t)}{d t}\right]
$$

where $e(t)=\left[\Delta P_{d}, \Delta V_{d}, \Delta \dot{V}\right]$ represents deviation value of position information between two neighboring sample instance, $T_{I}$ is the integral constant, $T_{D}$ is the differential constant, $K_{P}$ is the proportionality constant. Suffering from non-linearity and time-varying characteristics of unmanned helicopter, traditional PID controllers, which use constant parameter control law, hardly achieve better flight performance. To overcome this problem, the improved PID controller uses time-varying function to describe corresponding features of non-linearity and time varying features of the unmanned helicopter based on prior knowledge of noise. In the improved PID controller, the constant control law $K_{P}$ is replaced by $K_{P}(e, k)$ :

$$
K_{P}(e, k)= \begin{cases}\left(1-\left(1-C_{P \min }\right) e^{(-h \mid e)}\right) & |e(k)|>\mathrm{M} \\ K_{P_{0}} & |e(k)| \leq \mathrm{M}\end{cases}
$$

where $\mathrm{M}$ is error threshold, $K_{P_{0}}$ is the initial value of proportionality function, $\mathrm{h}$ is a constant coefficient. $C_{P \text { min }}$ is the minimum decay rate of $K_{P}$. Based on the control law of $K_{p}(e, k)$, proportional control action may be enhanced when the system error is big and that it may be weaken when the system error is small. By such improvements, the controller can quickly control the position of unmanned helicopter.

\subsection{Inner-loop Controller based on Back-stepping}

The main task for inner-loop controller is to design a suitable control law for attitude angle equation $\left(\dot{\eta}=H(\eta) \omega^{b}\right)$ to control attitude angle $\left(\eta_{d}=\left[\begin{array}{lll}\phi_{d} & \theta_{d} & \psi_{d}\end{array}\right]\right)$ stably. Under condition of low speed flight, $H(\eta)$ can be approximately assumed to be identity matrix. Denote the state variables as $X=\left[\begin{array}{llllll}x_{1} & x_{2} & x_{3} & x_{4} & x_{5} & x_{6}\end{array}\right]^{T}=\left[\begin{array}{llllll}\phi & \dot{\phi} & \theta & \dot{\theta} & \psi & \dot{\psi}\end{array}\right]^{T}$, based on (1) and (3) , the nonlinear system model of the small unmanned helicopter can be given as:

$$
\dot{X}=f(X, U, t)=\left[\begin{array}{c}
x_{2} \\
\left(\frac{I_{y y}-I_{y y}}{I_{x x}}\right) x_{4} x_{6}+A_{P}+\frac{\tau_{x}}{I_{x x}} \\
x_{4} \\
\left(\frac{I_{z z}-I_{x x}}{I_{y y}}\right) x_{4} x_{6}+A_{q}+\frac{\tau_{y}}{I_{y y}} \\
x_{6} \\
\left(\frac{I_{x x}-I_{y y}}{I_{z z}}\right) x_{4} x_{6}+A_{r}+\frac{\tau_{z}}{I_{z z}}
\end{array}\right]
$$

where $A_{p}=E_{p}+F_{\text {exp }}, A_{q}=E_{q}+F_{\text {extq }}$ and $A_{r}=E_{r}+F_{\text {extr }}\left(E_{p}, E_{q}, E_{r}\right.$ represent system error of inner-loop controller, $F_{\text {extp }}, F_{\text {extq }}, F_{\text {extr }}$ are the external disturbance.)

Defining Lyapunov function as

$V_{1}=\frac{1}{2} z_{1}^{2}+\frac{k_{i}}{2}\left[\int_{0}^{t} z_{1} d \tau\right]^{T}\left[\int z_{1} d \tau\right]$ 
where $z_{1}=x_{1}-x_{1 d}$ is the error of roll angle, $k_{i}$ is a positive constants. To improve accuracy of controller, we introduce angular velocity error $z_{2}=x_{2}-x_{2}^{c}$ into the corresponding attitude angle related Lyapunov function $V_{2}=V_{1}+\frac{1}{2} z_{2}^{2}$. To estimate the system error $\hat{A}_{p}$ correctly, a new Lyapunov function is defined as $V_{3}=V_{2}+\frac{1}{2 \gamma} \tilde{A}_{p}^{2}$. Note that the Lyapunov function $\dot{V}_{3} \leq 0$, which implies that the proposed controller may control the attitude angles stably. Similarly, $\psi$ and $\theta$ can also be controlled effectively.

\section{Numerical Results}

To evaluate performance of the proposed controller, we simulated a reference trajectory, which is defined as $P_{d}^{i}=[2 \cos (t / 3), 2 \sin (t / 3),-1-t], \psi_{d}=0$. The initial position of the unmanned helicopter is $\left(\begin{array}{lll}x & y & z\end{array}\right)=\left(\begin{array}{lll}1.2 & 0.5 & -1\end{array}\right) m$, the initial attitude angle is $\left(\begin{array}{lll}\phi_{d} & \theta_{d} & \psi_{d}\end{array}\right)=\left(\begin{array}{llll}0 & 0.5 & 0.5\end{array}\right) \mathrm{rad}$. In the 10th sample instance, the unmanned helicopter began to be disturbed by wind gusts with the speed of $1.5 \mathrm{~m} / \mathrm{s}$ simultaneously in $x, y, z$ directions respectively.

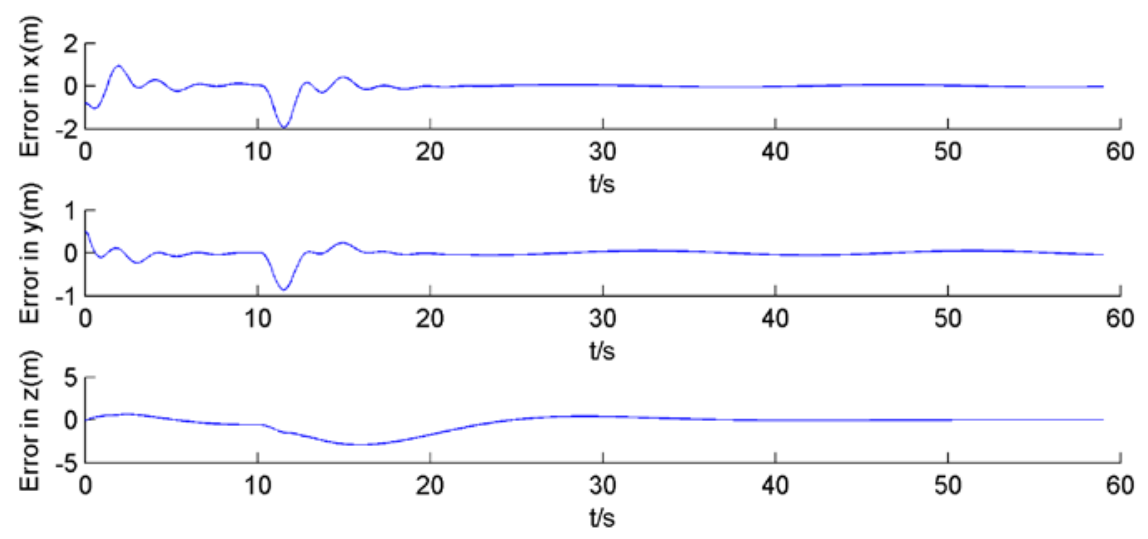

Fig. 1 Position error in $x, y, z$ direction respectively

As shown in Fig. 1, in the simulated experiment, we added different disturbances at $t=10 \mathrm{~s}$ and $t=15$ s respectively. Under different disturbances, the proposed controller can quickly and exactly control the trajectory. Thus, the proposed controller can control the unmanned helicopter at a safe range stably.
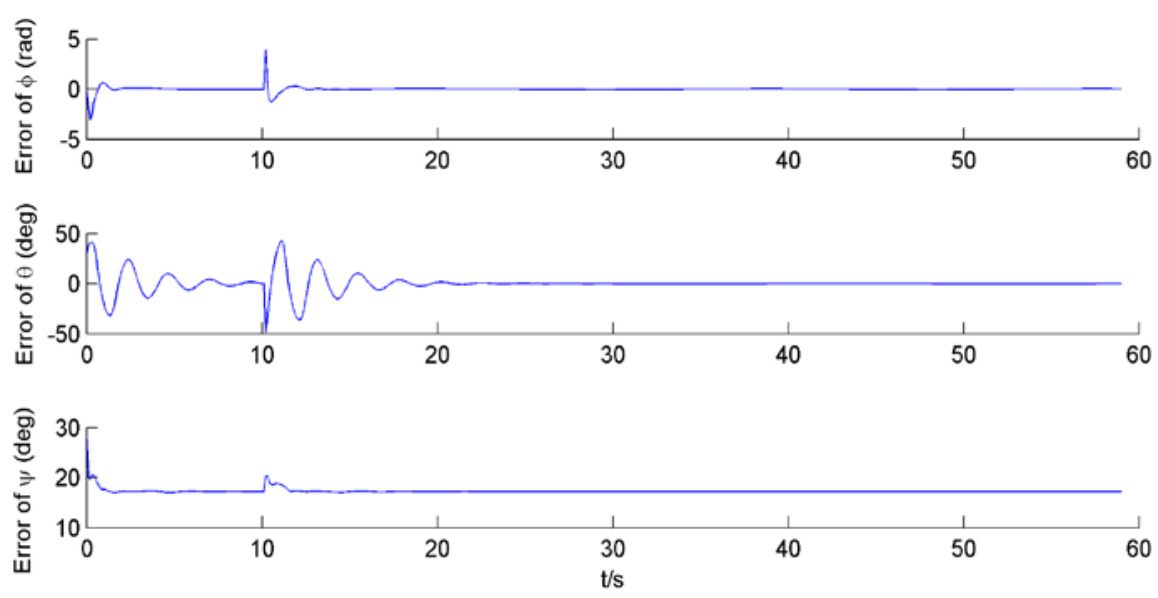

Fig. 2 Attitude angle error

Fig.2 shows the results of attitude angle trajectory based on the proposed controller. It can clearly be found that the initial disturbance (which is added at $t=10 \mathrm{~s}$ ) influenced the controller 
performance and caused system error. Fortunately, the proposed controller quickly returns the attitude angle to the preset point.

\section{Conclusions}

For characteristics of non-linearity and high order of unmanned helicopter system, an inner-outer loop controller is proposed in this paper. the proposed inner-outer loop controller can not only work under disturbance situations but also can be easily realized in practice by the simplification procedure in designing the non-linear system model. Numerical results on simulated data set validate that the proposed controller can quickly and stably control the unmanned helicopter under disturbance situation.

\section{Acknowledgements}

This work was financially supported by the Guangxi Natural Science Foundation (2016GXNSFBA380160), Guangxi Scientific Research Porjects (KY2015ZD143). NNSF (No.61072127), Characteristic Innovation Project of Guangdong Province(No.2015KTSCX143), Young innovative talents project of Guangdong Province(2015KQNCX165, 2015KQNCX172) and Youth Foundation of WuYi University (No.2013zk10).

\section{Reference:}

[1] K.P. Valavanis, Advances in Unmanned Aerial Vechicles State of the Art and the Road to Autonomy, Intelligent Systems, Control and Automation: Science and Engineering[M]. Berlin: Springer-Verlag, 2007

[2] M. B. Tischler, R. K. Remple. Aircraft and Rotorcraft System Identification: Engineering Methods With Flight Test Examples[M]. AIAA Education Series Reston: AIAA, 2006.

[3] Mettler B F, Tischler M B, et al. Attitude control optimization for a small-scale unmanned helicopter [C]. AIAA Guidance, Navigation and Control Conference, 2000.

[4] Chao-Yong Li, Wu-Xing Jing, Adaptive Backstepping-based Flight Control System using Integral Filters[J], Aerospace Science and Technology 13(2009) 105-113.

[5] Xiaohe, Liu. The causation of nonlinear system analyzing and controlling[M]. Peking: Tsinghua university press, April, 2008.

[6] Hassan K. Khalil. Nonlinear Systems(Third Edition). Publishing as Prentice Hall in 2002. 